Homology, Homotopy and Applications, vol.14(1), 2012, pp.159-180

\title{
COMPUTING BRAID GROUPS OF GRAPHS WITH APPLICATIONS TO ROBOT MOTION PLANNING
}

\author{
VITALIY KURLIN \\ (communicated by Robert Ghrist)
}

\begin{abstract}
An algorithm is designed to write down presentations of graph braid groups. Generators are represented in terms of actual motions of robots moving without collisions on a given connected graph. A key ingredient is a new motion planning algorithm whose complexity is linear in the number of edges and is quadratic in the number of robots. The computing algorithm implies that 2-point braid groups of all light planar graphs have presentations where all relators are commutators.
\end{abstract}

\section{Introduction}

\subsection{Brief summary}

This is a research on the interface between topology and graph theory with applications to motion planning algorithms in robotics. Consider moving objects as zero-size points that travel without collisions along fixed tracks that form a connected graph, say on a factory floor or road map. These objects will be called 'robots', although the reader may use a more neutral and abstract word like 'token'.

For practical reasons, discrete analogues of configuration spaces of graphs are studied. Then robots can not be very close to each other, at least one full open edge apart. This discrete approach reduces the motion planning of real (not zero-size) vehicles to combinatorial questions about ideal robots that move on a subdivided graph.

\subsection{Graphs and theirs configuration spaces}

Let us recall basic notions. A graph $G$ is a 1-dimensional finite $\mathrm{CW}$ complex whose 1-cells are supposed to be open. The 0-cells and open 1-cells are called vertices and edges, respectively. If the endpoints of an edge $e$ are the same, then $e$ is called a loop. A multiple edge is a collection of edges with the same distinct endpoints. The topological closure $\bar{e}$ of an edge $e$ is the edge $e$ itself with its endpoints.

The degree $\operatorname{deg} v$ of a vertex $v$ is the number of edges attached to $v$, i.e., a loop contributes 2 to the degree of its vertex. Vertices of degree 1 or 2 are hanging or trivial, respectively. Vertices of degree at least 3 are essential. A path (a cycle, respectively)

Received November 4, 2009, revised June 8, 2011; published on June 4, 2012.

2000 Mathematics Subject Classification: 57M05, 20F36, 05C25.

Key words and phrases: graph, braid group, configuration space, fundamental group, homotopy type, deformation retraction, collision free motion, planning algorithm, complexity, robotics.

Article available at http://intlpress.com/HHA/v14/n1/a8 and doi:10.4310/HHA.2012.v14.n1.a8

Copyright (C) 2012, International Press. Permission to copy for private use granted. 
of a length $k$ in $G$ is a subgraph that consists of $k$ edges and is homeomorphic to a segment (a circle, respectively). A tree is a connected graph without cycles.

The direct product $G^{n}=G \times \cdots \times G$ ( $n$ times) has the product structure of a 'cubical' complex such that each product $\bar{c}_{1} \times \cdots \times \bar{c}_{n}$ is isometric to a Euclidean cube $[0,1]^{k}$, where $\bar{c}_{i}$ is the topological closure of a cell of $G$. The dimension $k$ is the number of the cells $c_{i}$ that are edges of $G$. The diagonal of the product $G^{n}$ is

$$
\Delta\left(G^{n}\right)=\left\{\left(x_{1}, \ldots, x_{n}\right) \in G^{n} \mid x_{i}=x_{j} \text { for some } i \neq j\right\} .
$$

Definition 1.1. Let $G$ be a graph, $n$ be a positive integer. The ordered topological configuration space $\mathbf{O C}(G, n)$ of $n$ distinct robots in $G$ is $G^{n}-\Delta\left(G^{n}\right)$. The unordered topological configuration space $\mathbf{U C}(G, n)$ of $n$ indistinguishable robots in $G$ is the quotient of $\mathbf{O C}(G, n)$ by the action of the permutation group $S_{n}$ of $n$ robots.

The ordered topological space $\mathbf{O C}([0,1], 2)=\left\{(x, y) \in[0,1]^{2} \mid x \neq y\right\}$ is the unit square without its diagonal. This space is homotopy equivalent to a disjoint union of two points. Spaces $X, Y$ are homotopy equivalent if there are continuous maps

$$
f: X \rightarrow Y, \quad g: Y \rightarrow X \quad \text { such that } \quad g \circ f: X \rightarrow X, \quad f \circ g: Y \rightarrow Y
$$

can be connected with the identity maps $\operatorname{id}_{X}: X \rightarrow X, \operatorname{id}_{Y}: Y \rightarrow Y$, respectively, through continuous families of maps. In particular, $X$ is contractible if $X$ is homotopy equivalent to a point. A space $X$ can be homotopy equivalent to its subspace $Y$ by a deformation retraction that is a continuous family of maps $f_{t}: X \rightarrow X, t \in[0,1]$, such that $\left.f_{t}\right|_{Y}=\mathrm{id}_{Y}$, i.e., all $f_{t}$ are fixed on $Y, f_{0}=\operatorname{id}_{X}$ and $f_{1}(X)=Y$.

The unordered topological space $\mathbf{U C}([0,1], 2) \approx\left\{(x, y) \in[0,1]^{2} \mid x<y\right\}$ is contractible to a single point. More generally, OC $([0,1], n)$ has $n$ ! contractible connected components, while $\mathbf{U C}([0,1], n)$ deformation retracts to the standard configuration $x_{i}=(i-1) /(n-1), i=1, \ldots, n$, in $[0,1]$. If a connected graph $G$ has a vertex of degree at least 3 , then the configuration spaces $\mathbf{O C}(G, n), \mathbf{U C}(G, n)$ are path-connected. Swap robots $x, y$ near such a vertex as shown in Figure 1.

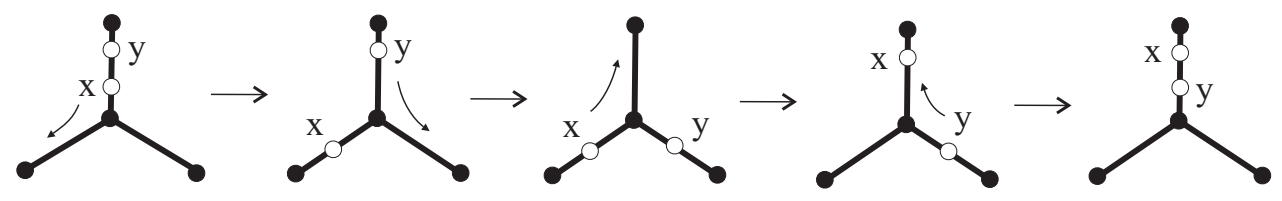

Figure 1: Swapping two robots $x, y$ without collisions on the tripod $T$

Definition 1.2. Given a connected graph $G$ having a vertex of degree at least 3 , the graph braid groups $\mathbf{P}(G, n)$ and $\mathbf{B}(G, n)$ are the fundamental groups $\pi_{1}(\mathbf{O C}(G, n))$ and $\pi_{1}(\mathbf{U C}(G, n))$, respectively, where arbitrary base points are fixed.

For the tripod $T$ in Figure 1, both configuration spaces $\mathbf{O C}(T, 2), \mathbf{U C}(T, 2)$ are homotopy equivalent to a circle; see Example 2.1, i.e., $\mathbf{B}(T, 2) \cong \mathbb{Z}, \mathbf{P}(T, 2) \cong \mathbb{Z}$, although $\mathbf{P}(T, 2)$ can be considered as an index 2 subgroup $2 \mathbb{Z}$ of $\mathbf{B}(T, 2) \cong \mathbb{Z}$.

Definition 1.3. The ordered discrete space $\operatorname{OD}(G, n)$ consists of all the products $\bar{c}_{1} \times \cdots \times \bar{c}_{n}$ such that each $c_{i}$ is a cell of $G$ and $\bar{c}_{i} \cap \bar{c}_{j}=\varnothing$ for $i \neq j$. The unordered discrete space $\mathbf{U D}(G, n)$ is the quotient of $\mathbf{O D}(G, n)$ by the action of $S_{n}$. 
The support $\operatorname{supp}(H)$ of a subset $H \subset G$ is the minimum union of closed cells containing $H$. For instance, the support of a vertex or an open edge coincides with its topological closure in the graph $G$. The support of a point interior to an open edge $e$ is $\bar{e}$, i.e., the edge $e$ with its endpoints. A configuration $\left(x_{1}, \ldots, x_{n}\right) \in G^{n}$ is safe if $\operatorname{supp}\left(x_{i}\right) \cap \sup \left(x_{j}\right)=\varnothing$ whenever $i \neq j$. Then the discrete configuration space $\mathbf{O D}(G, n)$ consists of all safe configurations:

$$
\mathbf{O D}(G, n)=\left\{\left(x_{1}, \ldots, x_{n}\right) \in G^{n} \mid \operatorname{supp}\left(x_{i}\right) \cap \operatorname{supp}\left(x_{j}\right)=\varnothing, i \neq j\right\} .
$$

A path in a graph $G$ is essential if the path connects distinct vertices of degrees not equal to 2 . A cycle in $G$ is essential if the cycle contains a vertex of a degree more than 2. Since only connected graphs are considered, a non-essential cycle coincides with the whole graph. Subdivision Theorem 1.4 provides sufficient conditions such that the configuration spaces $\mathbf{O C}(G, n), \mathbf{U C}(G, n)$ deformation retract to their discrete analogues $\mathbf{O D}(G, n), \mathbf{U D}(G, n)$, respectively. Then $\mathbf{B}(G, n) \cong \pi_{1}(\mathbf{U D}(G, n))$.

Theorem 1.4 ([1, Theorem 2.1]). Let $G$ be a connected graph, $n \geqslant 2$. The discrete spaces $\mathbf{O D}(G, n), \mathbf{U D}(G, n)$ are deformation retracts of the topological configuration spaces $\mathbf{O C}(G, n), \mathbf{U C}(g, n)$, respectively, if both conditions (1.4a) and (1.4b) hold:

(1.4a) Every essential path in $G$ has at least $n+1$ edges;

(1.4b) Every essential cycle in $G$ has at least $n+1$ edges.

The conditions above imply that $G$ has at least $n$ vertices, so $\mathbf{O D}(G, n) \neq \varnothing$. A strengthened version of Subdivision Theorem 1.4 for $n=2$ only requires that $G$ has no loops and multiple edges [1, Theorem 2.4]. The topological configuration spaces of two points on the Kuratowski graphs $K_{5}, K_{3,3}$ in Figure 2 deformation retract to their smaller discrete analogues. In $\mathbf{O D}\left(K_{5}, 2\right)$, if the first robot is moving along an edge $h \in K_{5}$, then the second robot can be only in the triangular cycle $C \subset$ $K_{5}-h$. In total, these ten triangular tubes $h \times C$ form the oriented surface of genus 6. Similarly, computing the Euler characteristic, we conclude that $\mathbf{O D}\left(K_{3,3}, 2\right)$ is the oriented surface of genus 4 . These are the only graphs without loops whose discrete configuration spaces $\mathbf{O D}(G, 2)$ are closed manifolds; see [1, Corollary 5.8].

Students of L. Sabalka [12] have extended Subdivision Theorem 1.4 for any $n>2$ to the optimal criterion where $n+1$ is replaced by $n-1$ in condition (1.4a).
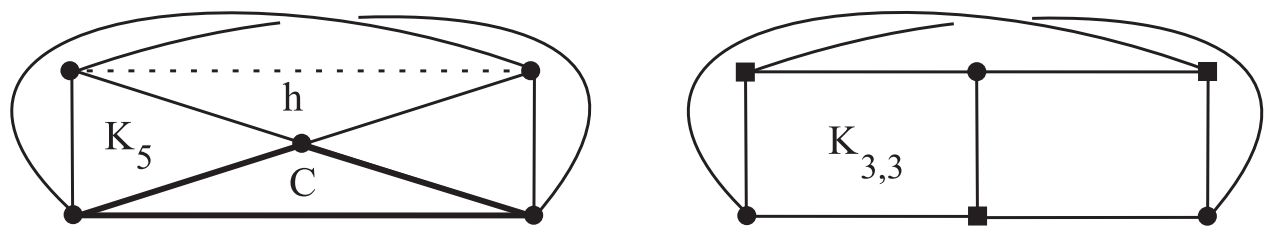

Figure 2: Kuratowski graphs $K_{5}$ and $K_{3,3}$

\subsection{Main results}

There are two known approaches to computing graph braid groups suggested by Abrams [1, Section 3.2] and by Farley and Sabalka [5, Theorem 5.3]. In the former approach any pure graph braid group splits as a graph of simpler groups, which gives 
a nice global structure of the group and proves that all graph braid groups are torsion free [1, Corollary 3.7 on p. 25]. The latter approach is based on the discrete Morse theory developed by Forman [7]. One writes presentations of graph braid groups by retracting a big discrete configuration space to a smaller subcomplex.

This paper proposes another local approach based on the classical Seifert-van Kampen Theorem 3.1. Presentations are computed step-by-step starting from simple graphs and adding edges one-by-one, which allows us to update growing networks in real-time. Resulting Algorithm 1.5 expresses generators of graph braid groups using actual motions of robots, i.e., as a list of positions at discrete time moments. The key ingredient is new motion planning Algorithm 4.3 to connect any configurations of $n$ robots. The computational complexity of Algorithm 4.3 is linear in the number of edges and is quadratic in the number of robots.

Algorithm 1.5. For a connected graph $G$, there is an algorithm to write down a presentation of the graph braid group $\mathbf{B}(G, n)$. Generators are represented by actual paths between configurations; see step-by-step instructions in Subsection 4.1.

According to [6, Theorem 5.6], the braid groups of planar graphs having only disjoint cycles have presentations where each relator is a commutator, not necessarily a commutator of generators. To demonstrate the power of Algorithm 1.5, this result is extended to a wider class of light planar graphs. A planar connected graph $G$ is called light if any cycle $C \subset G$ has an open edge $h$ such that all cycles from $G-\bar{h}$ do not meet $C$. Removing the closure $\bar{h}$ from $G$ is equivalent to removing the endpoints of $h$ and all open edges attached to the endpoints. Any loop or a multiple edge provides an edge $h$ satisfying the above condition. Figure 3 shows a non-light planar graph with four choices of a (dashed) edge $h$ and corresponding (fat) cycles from $G-\bar{h}$. Indeed, in each case the fat cycles from $G-\bar{h}$ meet any cycle $C$ containing $h$.
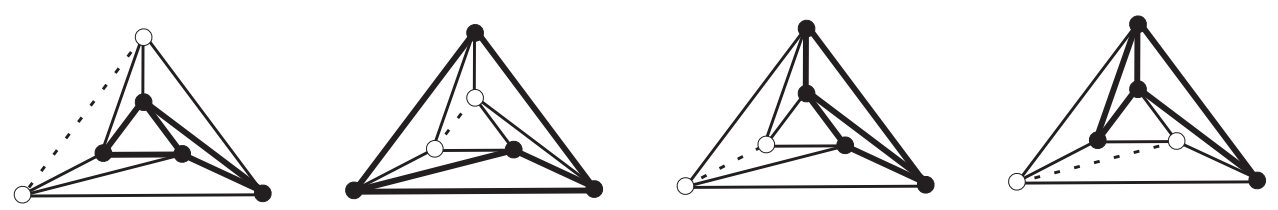

Figure 3: A non-light planar graph with four choices of a closed edge $\bar{h}$

Corollary 1.6. The braid group $\mathbf{B}(G, 2)$ of any light planar graph $G$ has a presentation where each relator is a commutator of motions along disjoint cycles.

A stronger version of Corollary 1.6 with a geometric description of generators and relators is given in Proposition 4.8 in the case of unordered robots.

\section{Outline}

Section 2 considers basic examples and recalls related results. Section 3 introduces the computing engine of Propositions 3.2, 3.4, 3.6 updating presentations of graph braid groups by adding edges one-by-one. Section 4 lists step-by-step instructions of Algorithm 1.5 computing graph braid groups. As an application, 2-point braid groups of light planar graphs are described in simple combinatorial terms. 


\section{Acknowledgements}

The author thanks the anonymous referee for helpful suggestions and Lucas Sabalka for his valuable corrections in Subsections 2.2, 3.1 and 4.2.

\section{Discrete configuration spaces of a graph}

In this section discrete configuration spaces are discussed in more detail. These spaces are recursively constructed in Lemmas 2.5 and 2.6. Further assume that the number of robots is $n \geqslant 2$.

\subsection{Configuration spaces of the tripod $T$}

This subsection describes configuration spaces of two points on the tripod $T$. The tripod $T$ consists of three hanging edges $e_{1}, e_{2}, e_{3}$ that are attached to the central vertex $v$; see Figure 4 .

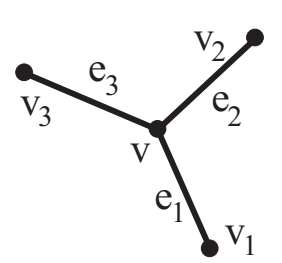

$\mathrm{T}$

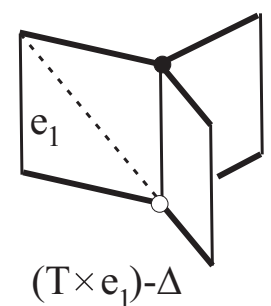

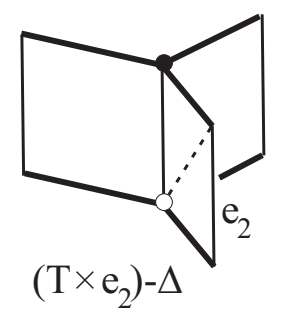

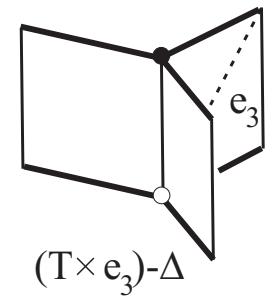

$\left(\mathrm{T} \times \mathrm{e}_{3}\right)-\Delta$

Figure 4: The tripod $T$ and $\left(T \times e_{1}\right)-\Delta,\left(T \times e_{2}\right)-\Delta,\left(T \times e_{3}\right)-\Delta$

Example 2.1. The ordered topological space $\mathbf{O C}(T, 2)$ is the union of three 3-page books $T \times e_{1}, T \times e_{2}, T \times e_{3}$ shown in the right-hand side pictures of Figure 4 without the diagonal $\Delta=\left\{(x, y) \in T^{2} \mid x \neq y\right\}$. Then $\mathbf{O C}(T, 2)$ consists of the six symmetric rectangles $e_{i} \times e_{j}(i \neq j)$ and six triangles from the squares $e_{i} \times e_{i}, i=1,2,3$, after removing their diagonals; see the left-hand side picture of Figure 5 and $[\mathbf{2}$, Example 6.26].

Example 2.2. The ordered topological space $\mathbf{O C}(T, 2)$ deformation retracts to the polygonal circle in the right-hand side picture of Figure 5. This circle is the ordered discrete space $\mathbf{O D}(T, 2)$ having 12 vertices $v_{i} \times v_{j}(i \neq j)$ and $v \times v_{i}, v_{i} \times v, i=$ $1,2,3$, symmetric under the permutation of factors. The unordered spaces $\mathbf{U C}(T, 2)$, $\mathbf{U D}(T, 2)$ are the quotients of the corresponding ordered spaces by the rotation through $\pi$ and are homeomorphic to $\mathbf{O C}(T, 2), \mathbf{O D}(T, 2)$, respectively. Hence the graph braid groups $\mathbf{B}(T, 2) \cong \mathbb{Z}, \mathbf{P}(T, 2) \cong \mathbb{Z}$ can be computed by using the simpler discrete spaces $\mathbf{U D}(T, 2), \mathbf{O D}(T, 2)$, which is reflected in Theorem 1.4.

\subsection{Recursive construction of discrete spaces}

This subsection explains recursive constructions of discrete configuration spaces. The constructions will be used in Section 3 to compute fundamental groups of the configuration spaces. 

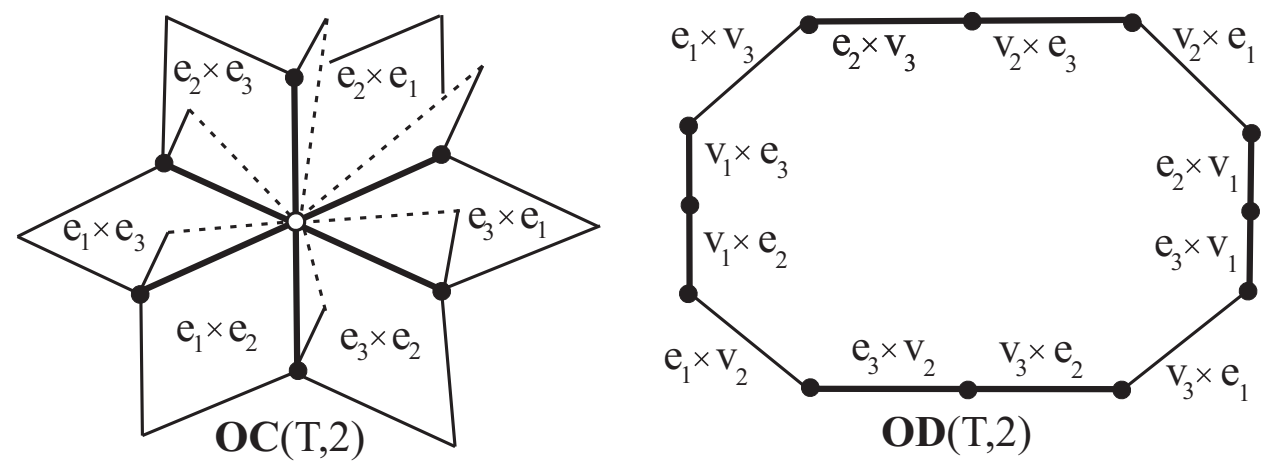

Figure 5: The ordered space $\mathbf{O C}(T, 2)$ and its discrete analogue $\mathbf{O D}(T, 2)$

Example 2.3. Let us show how to construct the ordered space $\mathbf{O D}(T, 2)$ adding the closed edge $\bar{e}_{1}$ to the subgraph $T-\left(e_{1} \cup v_{1}\right)=\bar{e}_{2} \cup \bar{e}_{3} \approx[0,1]$. If both robots $x, y$ are not in the open edge $e_{1}$, then $(x, y) \in \mathbf{O D}\left(T-e_{1}, 2\right)$, where $T-e_{1} \approx v_{1} \cup \bar{e}_{2} \cup \bar{e}_{3}$, i.e., either $x=v_{1}, y \in \bar{e}_{2} \cup \bar{e}_{3}$ (or vice versa) or $(x, y) \in \mathbf{O D}\left(\bar{e}_{2} \cup \bar{e}_{3}, 2\right)$. Since $x$ can not be close to $y$ by Definition 1.3 (e.g., if $x \in e_{1}$, then $y \in\left\{v_{2}, v_{3}\right\}$ ), then one has

$$
\mathbf{O D}(T, 2) \approx \mathbf{O D}\left(T-e_{1}, 2\right) \cup\left(\left\{v_{2}, v_{3}\right\} \times \bar{e}_{1}\right) \cup\left(\bar{e}_{1} \times\left\{v_{2}, v_{3}\right\}\right),
$$

where

$$
\mathbf{O D}\left(T-e_{1}, 2\right) \approx \mathbf{O D}\left(\bar{e}_{2} \cup \bar{e}_{3}, 2\right) \cup\left(v_{1} \times\left(\bar{e}_{2} \cup \bar{e}_{3}\right)\right) \cup\left(\left(\bar{e}_{2} \cup \bar{e}_{3}\right) \times v_{1}\right) .
$$

The subspace $\mathbf{O D}\left(T-e_{1}, 2\right)$ consists of four fat segments in the right-hand side picture of Figure 5, where the two horizontal segments represent $\mathbf{O D}\left(\bar{e}_{2} \cup \bar{e}_{3}, 2\right)$. Taking the quotient by swapping the robots, similarly decompose the unordered space

$$
\mathbf{U D}(T, 2) \approx \mathbf{U D}\left(\bar{e}_{2} \cup \bar{e}_{3}, 2\right) \cup\left(\left(\bar{e}_{2} \cup \bar{e}_{3}\right) \tilde{\times} v_{1}\right) \cup\left(\left\{v_{2}, v_{3}\right\} \tilde{\times} \bar{e}_{1}\right) .
$$

Here $A \tilde{\times} B$ denotes the quotient of $(A \times B) \cup(B \times A)$ over the symmetry that swaps the factors. The left-hand side picture of Figure 6 shows the subspace $\operatorname{UD}\left(\bar{e}_{2} \cup\right.$ $\left.\bar{e}_{3}, 2\right) \approx[0,1]$ and the whole space $\mathbf{U D}(T, 2) \approx S^{1}$. The segments $v_{2} \tilde{\times} \bar{e}_{1}$ and $v_{3} \tilde{\times} \bar{e}_{1}$ are glued at the endpoints $v_{2} \tilde{\times} v_{1}, v_{3} \tilde{\times} v_{1}$ and $v_{2} \tilde{\times} v, v_{3} \tilde{\times} v$, respectively.
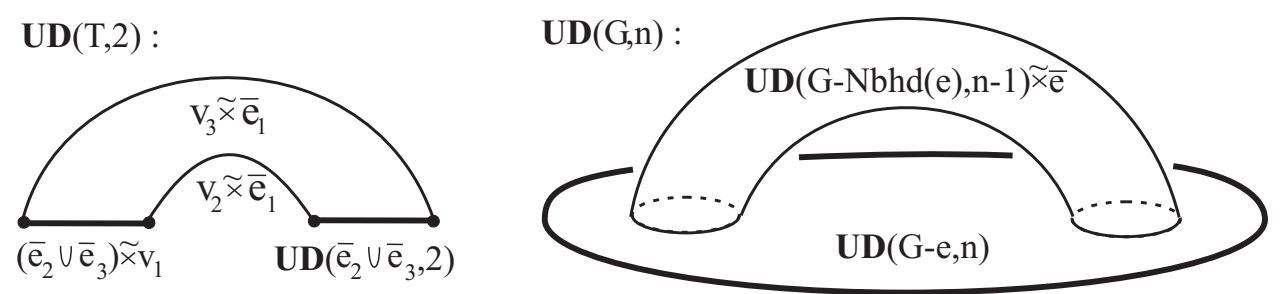

Figure 6: Attaching the cylinder in the recursive construction of $\mathbf{U D}(G, n)$

Example 2.3 motivates the following notion. The neighbourhood $\operatorname{Nbhd}(e)$ of an open edge $e \in G$ consists of $\bar{e}$ and all open edges attached to the endpoints of $e$. For 
instance, the complement to the neighbourhood $\operatorname{Nbhd}\left(e_{1}\right)$ in the tripod $T$ consists of the hanging vertices $v_{2}, v_{3}$; see the left-hand side picture of Figure 4.

Example 2.4. Extending the recursive idea of Example 2.3, let us construct the ordered 2-point space $\mathbf{O D}(G, 2)$ of any connected graph $G$. Fix an open edge $e \subset G$ with vertices $u, v$ and consider the case when one of the robots, say $y$, stays in $e$. Then $x \in G-\operatorname{Nbhd}(e)$ because $x$ can not be in the same edge $e$ and also in the edges adjacent to $e$. If both robots $x, y$ are not in $e$, then $(x, y)$ is in the smaller ordered space $\mathbf{O D}(G-e, 2)$. Then $\mathbf{O D}(G, 2)$ is a union of smaller subspaces:

$$
\mathbf{O D}(G, 2) \approx \mathbf{O D}(G-e, 2) \cup((G-\operatorname{Nbhd}(e)) \times \bar{e}) \cup(\bar{e} \times(G-\operatorname{Nbhd}(e))) .
$$

Here the first cylinder $(G-\operatorname{Nbhd}(e)) \times \bar{e}$ is glued to $\mathbf{O D}(G-e, 2)$ along the subgraphs $(G-\operatorname{Nbhd}(e)) \times u$ and $(G-\operatorname{Nbhd}(e)) \times v$. The second cylinder is symmetric to the first one. Taking the quotient over swapping the factors, one gets

$$
\mathbf{U D}(G, 2) \approx \mathbf{U D}(G-e, 2) \cup((G-\operatorname{Nbhd}(e)) \tilde{\times} \bar{e}),
$$

where $A \tilde{\times} B=((A \times B) \cup(B \times A)) / \sim$. Lemmas 2.5 and 2.6 are discrete analogues of Ghrist's construction of the ordered topological space $\mathbf{O C}(G, n)[\mathbf{8}$, Lemma 2.1].

Lemma 2.5. Let a connected graph $G$ have an open edge e with vertices $u, v$. Then the ordered discrete space $\mathbf{O D}(G, n)$ is homeomorphic to the union

$$
\mathbf{O D}(G, n) \approx \mathbf{O D}(G-e, n) \cup_{i=1}^{n}\left(\mathbf{O D}_{(i)}(G-\operatorname{Nbhd}(e), n-1) \times \bar{e}\right),
$$

where

$$
\mathbf{O D}_{(i)}(G-\operatorname{Nbhd}(e), n-1) \times \bar{e}=\left\{\mathbf{x} \in \mathbf{O D}(G, n) \mid x_{i} \in \bar{e}\right\}
$$

is glued to $\mathbf{O D}(G-e, n)$ along

$$
\mathbf{O D}_{(i)}(G-\operatorname{Nbhd}(e), n-1) \times u=\left\{\mathbf{x} \in \mathbf{O D}(G-\operatorname{Nbhd}(e), n) \mid x_{i}=u\right\}
$$

and

$$
\mathbf{O D}_{(i)}(G-\operatorname{Nbhd}(e), n-1) \times v=\left\{\mathbf{x} \in \mathbf{O D}(G-\operatorname{Nbhd}(e), n) \mid x_{i}=v\right\}, i=1, \ldots, n .
$$

Proof. In the space $\mathbf{O D}(G, n)$ of all safe configurations $\mathbf{x}=\left(x_{1}, \ldots, x_{n}\right)$ consider the smaller subspace $\mathbf{O D}(G-e, n)$, where $x_{i} \notin e$ for all $i=1, \ldots, n$. The complement $\mathbf{O D}(G, n)-\mathbf{O D}(G-e, n)$ consists of configurations with $x_{i} \in e$ for some $i$. By Definition 1.3 the other robots $x_{j} \notin \operatorname{Nbhd}(e)$ for $j \neq i$, i.e., the complement is

$$
\mathbf{O D}(G, n)-\mathbf{O D}(G-e, n) \approx \cup_{i=1}^{n} \mathbf{O D}_{(i)}(G-\operatorname{Nbhd}(e), n-1) \times e .
$$

The bases of each cylinder above are subspaces of the smaller configuration space:

$$
\mathbf{O D}_{(i)}(G-\operatorname{Nbhd}(e), n-1) \times u, \mathbf{O D}_{(i)}(G-\operatorname{Nbhd}(e), n-1) \times u \subset \mathbf{O D}_{(i)}(G-e, n) .
$$

The cylinder $\mathbf{O D}_{(i)}(G-\operatorname{Nbhd}(e), n-1) \times e$ represents motions when the $i$-th robot moves along $e$, while the other robots travel within $\mathbf{O D}(G-\operatorname{Nbhd}(e), n-1)$.

In Sections 3 and 4 the simpler unordered case is considered. For subspaces $A_{j}$ of the ordered space of $n_{j}$ robots, the direct product $A_{1} \times \cdots \times A_{k}$ lives in the ordered configuration space of $n=n_{1}+\cdots+n_{k}$ robots. The unordered configuration space of the same $n$ robots has no similar direct products. One can take the union of the 
products consisting of the factors $A_{1}, \ldots, A_{k}$ over all orders and then quotient the union by the permutation group $S_{n}$. The result can be denoted by $A_{1} \tilde{\times} \cdots \tilde{\times} A_{k}$.

Lemma 2.6. Let a connected graph $G$ have an open edge e with vertices $u, v$. Then the unordered discrete space $\mathbf{U D}(G, n)$ is homeomorphic to (see Figure 6)

$$
\mathbf{U D}(G, n) \approx \mathbf{U D}(G-e, n) \cup(\mathbf{U D}(G-\operatorname{Nbhd}(e), n-1) \tilde{\times} \bar{e}),
$$

where the subspace $\mathbf{U D}(G-\operatorname{Nbhd}(e), n-1) \tilde{\times} \bar{e}$ is glued to $\mathbf{U D}(G-e, n)$ along

$$
\mathbf{U D}(G-\operatorname{Nbhd}(e), n-1) \tilde{\times} u \quad \text { and } \quad \mathbf{U D}(G-\operatorname{Nbhd}(e), n-1) \tilde{\times} v .
$$

Proof. Quotient the ordered space from Lemma 2.5 by the permutation group $S_{n}$ :

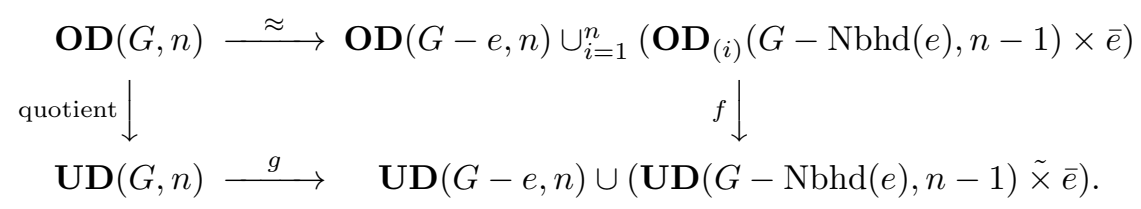

The natural projection $f$ above is continuous by the pasting lemma. The induced map $g$ is bijective and continuous by the universality property of quotients and defines a homeomorphism since $\mathbf{U D}(G, n)$ is compact, while its image is Hausdorff.

\subsection{Homotopy types of configuration spaces}

This subsection recalls general results on homotopy types of configuration spaces. A topological space $X$ is called aspherical or a $K(\pi, 1)$ space, if the space $X$ has a contractible universal cover, in particular $\pi_{i}(X)=0$ for $i>1$. A covering $p: Y \rightarrow X$ is universal, if the cover $Y$ is simply connected. Then the covering $p$ has the universal property that, for any covering $q: Z \rightarrow X$, there is another covering $Y \rightarrow Z$ whose composition with $q: Z \rightarrow X$ gives the original covering $p: Y \rightarrow X$.

Proposition 2.7 (Asphericity of configuration spaces, Ghrist [8, Corollary 2.4, Theorem 3.1] for topological spaces and Abrams [1, Section 3.2] for discrete spaces). Every component of $\mathbf{O C}(G, n), \mathbf{U C}(G, n), \mathbf{O D}(G, n), \mathbf{U D}(G, n)$ is aspherical.

Ghrist [8, Corollary 2.4, Theorem 3.1] proves the above result for the ordered topological space $\mathbf{O C}(G, n)$, which implies the same conclusion for $\mathbf{U C}(G, n)$, because the universal cover of a component of $\mathbf{U C}(G, n)$ is a universal cover of some component of $\mathbf{O C}(G, n)$ as mentioned by Abrams [1, the proof of Corollary 3.6].

Proposition 2.8 implies that the homotopy type of discrete spaces depends on the graph $G$, but not on the number $n$ of robots. It was proved by Ghrist $[\mathbf{8}$, Theorems 2.6 and 3.3] for $\mathbf{O C}(G, n)$. The result easily extends to the unordered case.

The circle $S^{1}$ is excluded below, because its unordered space $\mathbf{U C}\left(S^{1}, n\right)$ is homotopically equivalent to a circle, while $\mathbf{O C}\left(S^{1}, n\right)$ deformation retracts to a disjoint union of $(n-1)$ ! circles indexed by permutations of $n$ robots up to cyclic shifts.

Proposition 2.8 (Homotopy type of topological configuration spaces). If a connected graph $G \not \approx S^{1}$ has exactly $m$ essential vertices (of degree at least 3), then $\mathbf{O C}(G, n)$ and $\mathbf{U C}(G, n)$ deformation retract to m-dimensional complexes.

For instance, the configuration spaces of two robots in the tripod $T$ having a single essential vertex deformation retract to a 1-dimensional circle; see Examples 2.1, 2.2. 


\section{Fundamental groups of unordered discrete spaces}

In this section one computes graph braid groups. Namely, one shows how their presentations change by Seifert-van Kampen Theorem 3.1 after adding new edges to a graph. Let $X, Y$ be open path-connected subsets of $X \cup Y$ such that $X \cap Y \neq \varnothing$ is also path-connected. If $X, Y$ are not open in $X \cup Y$, then they usually can be replaced by their open neighbourhoods that deformation retract to $X, Y$, respectively. Assume that $X, Y, X \cap Y, X \cup Y$ have a common base point. If $\boldsymbol{\alpha}$ is a finite vector of elements, then a group presentation has the form $\langle\boldsymbol{\alpha} \mid \boldsymbol{\rho}\rangle$, where the relator $\boldsymbol{\rho}$ (a vector of words in the alphabet $\boldsymbol{\alpha}$ ) denotes the vector relation $\boldsymbol{\rho}=1$. A practical reformulation of the Seifert-van Kampen Theorem is below.

Theorem 3.1 (Seifert-van Kampen Theorem [3, Theorem 3.6 on p. 71]). If presentations $\pi_{1}(X) \cong\langle\boldsymbol{\beta} \mid \boldsymbol{\lambda}\rangle, \pi_{1}(Y) \cong\langle\boldsymbol{\gamma} \mid \boldsymbol{\mu}\rangle$ are given and $\pi_{1}(X \cap Y)$ is generated by (a vector of) words $\boldsymbol{\alpha}$, then the group $\pi_{1}(X \cup Y)$ has the presentation $\pi_{1}(X \cup Y) \cong$ $\left\langle\boldsymbol{\beta}, \boldsymbol{\gamma} \mid \boldsymbol{\lambda}, \boldsymbol{\mu}, \boldsymbol{\alpha}_{X}=\boldsymbol{\alpha}_{Y}\right\rangle$, where $\boldsymbol{\alpha}_{X}, \boldsymbol{\alpha}_{Y}$ are obtained from the words $\boldsymbol{\alpha}$ by rewriting the words $\boldsymbol{\alpha}$ in the alphabets $\boldsymbol{\beta}, \boldsymbol{\gamma}$, respectively.

As an example, consider the 2-dimensional torus $X \cup Y$, where $X$ is the complement to a closed disk $D$, while $Y$ is a open neighbourhood of $D$, i.e., $X \cap Y$ is an annulus. Then $X$ is homotopically equivalent to a wedge of two circles, i.e., $\pi_{1}(X) \cong\{\alpha, \beta \mid\}$ is free, $\pi_{1}(Y) \cong\langle\mid\rangle$ is trivial and $\pi_{1}(X \cap Y) \cong \mathbb{Z}$, hence

$$
\pi_{1}(X \cup Y) \cong\left\{\alpha, \beta \mid \alpha \beta \alpha^{-1} \beta^{-1}\right\}
$$

as $\alpha \beta \alpha^{-1} \beta^{-1}$ represents the boundary of $D$.

Presentations of the groups $\pi_{1}(\mathbf{U D}(G, n)) \cong \mathbf{B}(G, n)$ will be written down step-bystep by adding edges to the graph and by monitoring changes in the presentations. The base of our recursive computation is the contractible space $\mathbf{U D}([0,1], n)$ of $n$ robots in a segment whose fundamental group is trivial.

In Proposition 3.2 one glues a hanging edge to a vertex of degree at least 2, e.g., to an internal vertex of $[0,1]$, which may create an essential vertex (of degree at least 3). In Proposition 3.4 one adds a hanging edge to a hanging vertex of degree 1, which does not create an essential vertex. In Example 3.5 and Proposition 3.6 one attaches an edge creating cycles. Algorithm 1.5 computing graph braid groups is essentially based on Propositions 3.2, 3.4, 3.6. Each step shows how a group presentation is gradually becoming more complicated.

\subsection{Adding a hanging edge in the unordered case}

Here one shows how a braid group of a graph changes after adding a hanging edge. In a graph $H$ choose a hanging (open) edge $e \subset H$ attached to a hanging vertex $u$ and a vertex $v$ of degree at least 3 . If the vertex $v$ has degree $\operatorname{deg} v$, then $H-\operatorname{Nbhd}(e)$ consists of $k=\operatorname{deg} v-1$ disjoint subgraphs $H_{1}, \ldots, H_{k}$. If an edge $e_{j} \neq e$ attached at $v$ is also hanging, then the corresponding graph $H_{j}$ is the other endpoint of $e_{j}$.

In $\mathbf{U D}(H, n)$ if one robot is in $e$, then the remaining $n-1$ unordered robots from $H-\operatorname{Nbhd}(e)$ split into $k$ ordered subsets having $j_{1}, \ldots, j_{k}$ robots in the subgraphs $H_{1}, \ldots, H_{k}$ respectively, so $j_{1}+\cdots+j_{k}=n-1$. For an ordered set $J=\left(j_{1}, \ldots, j_{k}\right)$ 
of non-negative integer indices such that $j_{1}+\cdots+j_{k}=n-1$, let

$$
\mathbf{U D}_{J}(H-\operatorname{Nbhd}(e), n-1)
$$

be the quotient of

$$
\mathbf{U D}\left(H_{1}, j_{1}\right) \times \cdots \times \mathbf{U D}\left(H_{k}, j_{k}\right)
$$

by the action of the permutation group $S_{n-1}$.

$\mathbf{U D}(H-\operatorname{Nbhd}(e), n-1)$ splits into the subspaces $\mathbf{U D}_{J}(H-\operatorname{Nbhd}(e), n-1)$ over all $J=\left(j_{1}, \ldots, j_{k}\right)$ with ordered non-negative integers such that $j_{1}+\cdots+j_{k}=$ $n-1$. For $n=2$, the index $J$ degenerates to a single index $j=1, \ldots, \operatorname{deg} v-1$ of the subgraph $H_{j}$ containing the only remaining robot. Fix base points:

$$
a \in \mathbf{U D}(H-(e \cup u), n), \quad c_{J} \in \mathbf{U D}_{J}(H-\operatorname{Nbhd}(e), n-1) .
$$

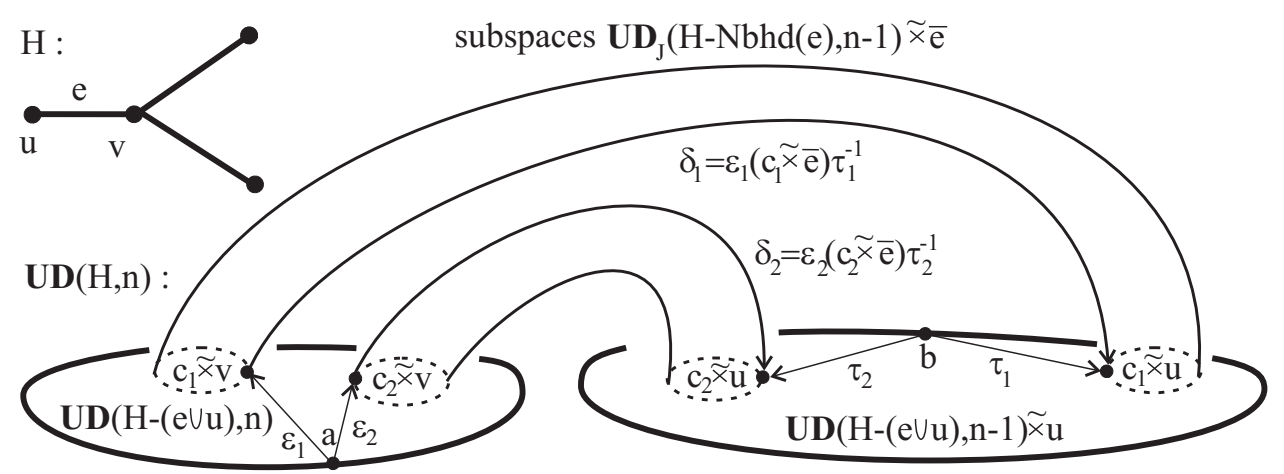

Figure 7: Adding a hanging edge $e$ to a non-hanging vertex $v$

Figure 7 shows two spaces of the form $\mathbf{U D}_{J}(H-\operatorname{Nbhd}(e), n-1) \tilde{x} \bar{e}$ indexed for simplicity by 1 and 2 as in the case $n=2$. Fix a point

$$
b \in \mathbf{U D}_{J}(H-(e \cup u), n-1) \tilde{\times} u,
$$

which can be chosen as $c_{1} \tilde{\times} u$, where $c_{1}$ is any fixed base point among $c_{J}$ 's. In $\mathbf{U D}(H-\operatorname{Nbhd}(e), n-1)$ find a path $\varepsilon_{J}$ from $a$ to $c_{J} \tilde{\times} v$, a path $\tau_{J}$ from $b$ to $c_{J} \times u$ for all $J=\left(j_{1}, \ldots, j_{k}\right), k=\operatorname{deg} v-1$. The configurations $c_{J} \tilde{\times} u, c_{J} \tilde{\times} v$ are connected by the motion $\left(c_{J} \tilde{\times} \bar{e}\right)$ when $n-1$ robots stay fixed at $c_{J} \in \mathbf{U D}(H-\operatorname{Nbhd}(e), n-1)$ and one robot moves along $\bar{e}$; see Figure 7 . Adding $\varepsilon_{J}, \tau_{J}^{-1}$ at the start and end of the motion $\left(c_{J} \tilde{\times} \bar{e}\right)$, respectively, one gets the paths $\delta_{J}$ going from $a$ to $b$ in $\mathbf{U D}(H, n)$.

Let us make some conventions. For a loop $\beta \subset \mathbf{U D}(H-(e \cup u), n-1)$ representing a motion of $n-1$ robots, the loop $\left(\beta\left\{x_{n}=u\right\}\right) \subset \mathbf{U D}(H-(e \cup u), n-1) \tilde{\times} u$ denotes the motion when $n-1$ robots follow $\beta$ and one robot is fixed at $u$. The index $n$ is only a part of the notation $\left(\beta\left\{x_{n}=u\right\}\right)$ since the robots are not ordered. The subspaces $\mathbf{U D}_{J}(H-\operatorname{Nbhd}(e), n-1)$ can also be disconnected, at least for $n=2$. So $\pi_{1}\left(\mathbf{U D}_{J}(H-\operatorname{Nbhd}(e), n-1)\right)$ is interpreted as a formal union of presentations for the fundamental groups of the connected components from $\mathbf{U D}_{J}(H-\operatorname{Nbhd}(e), n-$ 1). For instance, a generator of $\pi_{1}\left(\mathbf{U D}_{J}(H-\operatorname{Nbhd}(e), n-1)\right)$ for $J=\left(j_{1}, \ldots, j_{k}\right)$ means a motion when $j_{1}$ robots complete a loop in the subgraph $H_{1}$, other $j_{2}$ robots complete a loop in the subgraph $H_{2}$ disjoint from $H_{1}$ etc. 
Proposition 3.2 (Adding a hanging edge $e$ to a non-hanging vertex $v$ ). In the notations above and for presentations

$$
\pi_{1}(\mathbf{U D}(H-(e \cup u), n))=\langle\boldsymbol{\alpha} \mid \boldsymbol{\rho}\rangle
$$

and

$$
\begin{aligned}
\pi_{1}(\mathbf{U D}(H-(e \cup u), n-1)) & =\langle\boldsymbol{\beta} \mid \boldsymbol{\lambda}\rangle, \\
\pi_{1}\left(\mathbf{U D}_{J}(H-\operatorname{Nbhd}(e), n-1)\right) & =\left\langle\boldsymbol{\gamma}_{J} \mid \boldsymbol{\mu}_{J}\right\rangle,
\end{aligned}
$$

the group $\pi_{1}(\mathbf{U D}(H, n))$ is generated by $\boldsymbol{\alpha}, \delta_{1}\left(\boldsymbol{\beta}\left\{x_{n}=u\right\}\right) \delta_{1}^{-1}, \delta_{1} \delta_{J}^{-1}$, subject to $\boldsymbol{\rho}=1, \delta_{1}\left(\boldsymbol{\lambda}\left\{x_{n}=u\right\}\right) \delta_{1}^{-1}=1,\left(\gamma_{J}\left\{x_{n}=v\right\}\right)=\delta_{1}\left(\gamma_{J}\left\{x_{n}=u\right\}\right) \delta_{1}^{-1}$.

Proof. By the recursive construction from Lemma 2.6 one has

$$
\mathbf{U D}(H, n) \approx \mathbf{U D}(H-e, n) \cup(\mathbf{U D}(H-\operatorname{Nbhd}(e), n-1) \tilde{\times} \bar{e}) .
$$

Since $H-e$ splits into the vertex $u$ and the remaining subgraph $H-(e \cup u)$, then the space $\mathbf{U D}(H-e, n)$ splits into the subspaces $\mathbf{U D}(H-(e \cup u), n)$, where all robots are in $H-(e \cup u)$, and $\mathbf{U D}(H-(e \cup u), n-1) \tilde{\times} u$, where one robot is at $u$.

The non-connected subspace $\mathbf{U D}(H-\operatorname{Nbhd}(e), n-1) \tilde{\times} \bar{e}$ splits into the subspaces $\mathbf{U D}_{J}(H-\operatorname{Nbhd}(e), n-1) \tilde{\times} \bar{e}$ connecting the subspaces

$$
\mathbf{U D}(H-(e \cup u), n) \quad \text { and } \quad \mathbf{U D}(H-(e \cup u), n-1) \tilde{x} u .
$$

Indeed, the complement $H-\operatorname{Nbhd}(e)$ is obtained from $H$ by removing $u, v$ and all open edges attached to the vertex $v$ of degree $\operatorname{deg} v$.

Add the subspaces $\mathbf{U D}_{J}(H-\operatorname{Nbhd}(e), n-1) \tilde{x} \bar{e}$ over all $J=\left\{j_{1}, \ldots, j_{k}\right\}$ to $\mathbf{U D}(H-(e \cup u), n)$. The group $\pi_{1}(\mathbf{U D}(H-(e \cup u), n))$ is not affected. Actually, the added subspaces deformation retract to $\mathbf{U D}_{J}(H-\operatorname{Nbhd}(e), n-1) \times v$. This deformation is represented by a gradual movement of one robot along $\bar{e}$ towards $v$.

To apply Seifert-van Kampen Theorem 3.1 correctly, add all the paths $\delta_{J}$ to the resulting union above to get the new generators $\delta_{1} \delta_{J}^{-1}$; see Figure 7 . Here 1 denotes one fixed multiple index $J$ for simplicity, e.g., $1=(n-1,0, \ldots, 0)$.

Consider the space $\mathbf{U D}(H-(e \cup u), n-1) \tilde{\times} u$ as a subspace of $\mathbf{U D}(H, n)$. Formally a loop $\beta \in \pi_{1}(\mathbf{U D}(H-(e \cup u), n-1))$ becomes the loop $\left(\beta\left\{x_{n}=u\right\}\right)$ from $\pi_{1}(\mathbf{U D}(H-(e \cup u), n-1) \tilde{x} u)$, where one robot remains fixed at $u$. The same argument applies to the relator $\boldsymbol{\lambda}$. No other relations appear as the intersection of $\cup_{J} \delta_{J}$, and $\mathbf{U D}(H-(e \cup u), n) \cup_{J}\left(\mathbf{U D}_{J}(H-\operatorname{Nbhd}(e), n-1) \tilde{x} \bar{e}\right)$ contracts to $a$.

Take the union with the subspace $\mathbf{U D}(H-(e \cup u), n-1) \tilde{x} u$. So one adds the generators and relations of $\pi_{1}(\mathbf{U D}(H-(e \cup u), n-1))=\langle\boldsymbol{\beta} \mid \boldsymbol{\lambda}\rangle$. The intersection deformation retracts to the wedge of $\mathbf{U D}_{J}(H-\operatorname{Nbhd}(e), n-1) \tilde{\times} u$ over all $J$. Then each generator $\gamma_{J}$ gives a relation between the words representing $\left(\gamma_{J}\left\{x_{n}=v\right\}\right)$ in the spaces $\mathbf{U D}(H-(e \cup u), n)$ and $\mathbf{U D}(H-(e \cup u), n-1) \tilde{\times} u$. In the latter space the loop can be conjugated by $\delta_{1}$, which replaces $b$ by the point $a \in \mathbf{U D}(H, n)$.

In Proposition 3.2 the loops $\delta_{1}\left(\gamma_{J}\left\{x_{n}=u\right\}\right) \delta_{1}^{-1}$ live in $\mathbf{U D}(H-(e \cup u), n)$ with the base point $a$ and can be expressed in terms of the generators $\delta_{1}\left(\boldsymbol{\beta}\left\{x_{n}=u\right\}\right) \delta_{1}^{-1}$. So the last equality in the presentation is a valid relation between new generators. In the case $n=2$ the multiple index $J$ degenerates to a single index $j=1, \ldots, \operatorname{deg} v-1$. So one adds exactly $\operatorname{deg} v-2$ new generators of the form $\delta_{1} \delta_{j}^{-1}, j=2, \ldots, \operatorname{deg} v-1$. 


\subsection{Stretching a hanging edge in the unordered case}

This subsection shows how the presentation of a braid group of a tree changes after stretching a hanging edge of a tree. First consider the degenerate case of stretching a hanging edge $e$ of the tripod $T$ in the top left picture of Figure 8 .

Example 3.3. Let $H$ be the tree obtained by adding a hanging edge $g$ to the hanging vertex $u$ of the tripod $T$ in the top left picture of Figure 8. Namely, $T=H-(g \cup s)$, where $s$ is the only hanging vertex of $g$ in the tree $H$. The complement $F=H-$ $\operatorname{Nbhd}(g)$ consists of two hanging edges distinct from $e$ and meeting at the centre $v$ of the tripod $T$. Let us compute the braid group $\mathbf{B}(H, 2)$ by using $\mathbf{B}(T, 2) \cong \mathbb{Z}$ from Example 2.2. By Lemma 2.6 the unordered space $\mathbf{U D}(H, 2)$ has the form

$$
\mathbf{U D}(H, 2) \approx \mathbf{U D}(H-g, 2) \cup(F \tilde{\times} \bar{g}) \approx \mathbf{U D}(T, 2) \cup(T \tilde{\times} s) \cup(F \tilde{\times} \bar{g}) .
$$

Here the two components of $\mathbf{U D}(H-g, 2)$ are connected by the band $F \tilde{\times} \bar{g}$. First apply Seifert-van Kampen Theorem 3.1 to the union $\mathbf{U D}(T, 2) \cup(F \tilde{\times} \bar{g})$. Then the fundamental group is unchanged, i.e., it is isomorphic to $\mathbf{B}(T, 2) \cong \mathbb{Z}$. Indeed, the union deformation retracts to $\mathbf{U D}(T, 2)$. Then apply the same trick taking the union with $T \tilde{\times} s$. So one gets $\mathbf{B}(H, 2) \cong \mathbb{Z}$ for the same reasons.

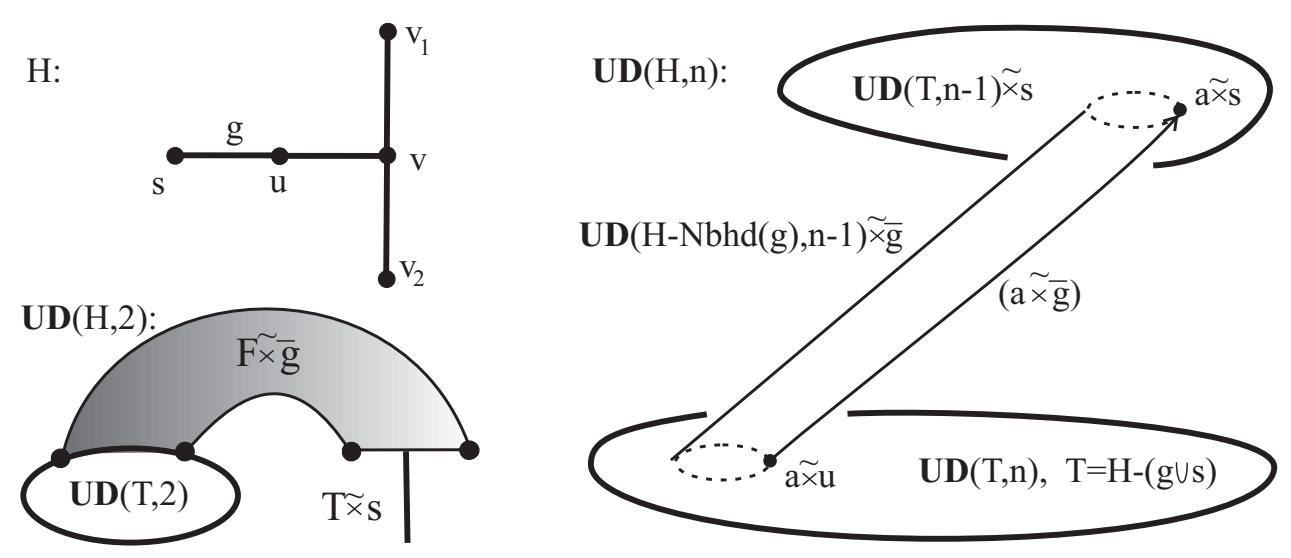

Figure 8: Stretching a hanging edge in a tree $H$

Proposition 3.4 below extends Example 3.3 to a general tree $H$. Choose an (open) edge $g \subset H$ with a hanging vertex $s$ and vertex $u$ of degree 2. Fix a base point:

$$
a \in \mathbf{U D}(H-\operatorname{Nbhd}(g), n-1) \subset \mathbf{U D}(H-(g \cup s), n-1) .
$$

Denote by $(a \tilde{\times} \bar{g})$ the motion from $a \tilde{\times} u$ to $a \tilde{\times} s$ in the space $\mathbf{U D}(H, n)$, when $n-1$ robots stay fixed at $a$, while one robot moves along $\bar{g}$; see the right picture of Figure 8. Then, for a loop $\gamma \in \pi_{1}(\mathbf{U D}(H-\operatorname{Nbhd}(g), n-1))$, both loops $\left(\gamma\left\{x_{n}=u\right\}\right)$ and $(a \tilde{\times} \bar{g})^{-1}\left(\gamma\left\{x_{n}=s\right\}\right)(a \tilde{\times} \bar{g})$ pass through the base point $a \tilde{\times} u \in \mathbf{U D}(H, n)$. 
Proposition 3.4 (Stretching a hanging edge). In the notations above and for presentations $\pi_{1}(\mathbf{U D}(H-(g \cup s), n))=\langle\boldsymbol{\alpha} \mid \boldsymbol{\rho}\rangle$ and

$$
\pi_{1}(\mathbf{U D}(H-(g \cup s), n-1))=\langle\boldsymbol{\beta} \mid \boldsymbol{\lambda}\rangle, \pi_{1}(\mathbf{U D}(H-\operatorname{Nbhd}(g), n-1))=\langle\boldsymbol{\gamma} \mid \boldsymbol{\mu}\rangle,
$$

$\pi_{1}(\mathbf{U D}(H, n))$ is generated by $\boldsymbol{\alpha},(a \tilde{\times} \bar{g})\left(\boldsymbol{\beta}\left\{x_{n}=s\right\}\right)(a \tilde{\times} \bar{g})^{-1}$ subject to $\boldsymbol{\rho}=1$,

$$
(a \tilde{\times} \bar{g})\left(\boldsymbol{\lambda}\left\{x_{n}=s\right\}\right)(a \tilde{\times} \bar{g})^{-1}=1,\left(\gamma\left\{x_{n}=u\right\}\right)=(a \tilde{\times} \bar{g})\left(\gamma\left\{x_{n}=s\right\}\right)(a \tilde{\times} \bar{g})^{-1} .
$$

Proof. By the recursive construction from Lemma 2.6 one has

$$
\mathbf{U D}(H, n) \approx \mathbf{U D}(H-g, n) \cup(\mathbf{U D}(H-\operatorname{Nbhd}(g), n-1) \tilde{\times} \bar{g}) .
$$

Here the subspace $\mathbf{U D}(H-\operatorname{Nbhd}(g), n-1) \tilde{\times} \bar{g}$ is glued to $\mathbf{U D}(H-g, n)$ along $\mathbf{U D}(H-\operatorname{Nbhd}(g), n-1) \tilde{\times} s$ and $\mathbf{U D}(H-\operatorname{Nbhd}(g), n-1) \tilde{\times} u$. Since $g$ is hanging, then $H-\operatorname{Nbhd}(g)$ has the two components: the hanging vertex $s$ and the remaining tree $T=H-(g \cup s)$, hence $\mathbf{U D}(H-g, n) \approx \mathbf{U D}(T, n) \cup(\mathbf{U D}(T, n-1) \tilde{\times} s)$.

Since the edge $e$ is hanging in $H-(g \cup s)$ before stretching, then the complement $H-\operatorname{Nbhd}(g)$ and the space $\mathbf{U D}(H-\operatorname{Nbhd}(g), n-1) \tilde{\times} \bar{g}$ are connected. Adding $\mathbf{U D}(H-\operatorname{Nbhd}(g), n-1) \tilde{\times} \bar{g}$ to $\mathbf{U D}(T, n)$ does not change the presentation. Indeed, the added subspace deformation retracts to $\mathbf{U D}(H-\operatorname{Nbhd}(g), n-1) \tilde{\times} u$.

Add $\mathbf{U D}(T, n-1) \tilde{\times} s$ meeting the union along $\mathbf{U D}(H-\operatorname{Nbhd}(g), n-1) \tilde{\times} s$. By Seifert-van Kampen Theorem 3.1, to get a presentation of $\pi_{1}(\mathbf{U D}(H, n))$ with the base point $a \tilde{\times} u$, one adds the generators $(a \tilde{\times} \bar{g})\left(\boldsymbol{\beta}\left\{x_{n}=s\right\}\right)(a \tilde{\times} \bar{g})^{-1}$ and relations $(a \tilde{\times} \bar{g})\left(\boldsymbol{\lambda}\left\{x_{n}=s\right\}\right)(a \tilde{\times} \bar{g})^{-1}$ coming from the group $\pi_{1}(\mathbf{U D}(T, n-1))$. Add the new relations $\left(\gamma\left\{x_{n}=u\right\}\right)=(a \tilde{\times} \bar{g})\left(\gamma\left\{x_{n}=s\right\}\right)(a \tilde{\times} \bar{g})^{-1}$ saying that the generators of the group $\pi_{1}(\mathbf{U D}(H-\operatorname{Nbhd}(g), n-1))$ after adding the stationary robot become homotopic through the subspace $\mathbf{U D}(H-\operatorname{Nbhd}(g), n-1) \tilde{\times} \bar{g}$.

\section{3. $\quad$ Creating cycles in the unordered case}

This subsection extends computations to graphs containing cycles. First let us show how the braid group changes if an edge is added at two vertices of a tripod.

Example 3.5. Let $G$ be the graph obtained from the tripod $T$ in the top left picture of Figure 9 by adding the edge $h$ at the vertices $r, w$. By Lemma 2.6 one has

$$
\mathbf{U D}(G, 2) \approx \mathbf{U D}(G-h, 2) \cup((G-\operatorname{Nbhd}(h)) \tilde{\times} \bar{e}) \approx \mathbf{U D}(T, 2) \cup(\bar{e} \tilde{\times} \bar{h}) .
$$

Geometrically the band $\bar{e} \tilde{\times} \bar{h}$ is glued to the hexagon $\mathbf{U D}(T, 2)$ as shown in the bottom left picture of Figure 9. To compute the graph braid group $\mathbf{B}(G, 2)$, first add to the band $\bar{e} \tilde{\times} \bar{h}$ the motions $\varepsilon, \tau \subset \mathbf{U D}(T, 2)$ connecting the base configuration $u \tilde{\times} v$ to $u \tilde{\times} r, u \tilde{\times} w$, respectively. This adds a generator to the trivial fundamental group of the contractible band $\bar{e} \tilde{x} \bar{h}$. Second, add the union $(\bar{e} \tilde{\times} \bar{h}) \cup(\varepsilon \cup \tau)$ to $\mathbf{U D}(T, 2)$, which gives $\mathbf{U D}(G, 2)$. The intersection of the spaces attached above has the form $(\bar{e} \tilde{\times} r) \cup(u \tilde{\times} \bar{h}) \cup(\bar{e} \tilde{\times} w)$ and is contractible. Hence $\mathbf{B}(G, 2) \cong F_{2}$ is the free group with two generators as the free product of $\mathbf{B}(T, 2) \cong \mathbb{Z}$ and $\pi_{1}((\bar{e} \tilde{\times} \bar{h}) \cup \varepsilon \cup \tau) \cong \mathbb{Z}$.

Proposition 3.6 extends Example 3.5 to a general graph excluding the case $G \approx$ $S^{1}$. Choose an (open) edge $h \subset G$ with vertices $r, w$ such that $G-h$ is connected. Let $G-\operatorname{Nbhd}(h)$ consist of $k$ connected components. Then $\mathbf{U D}(G-\operatorname{Nbhd}(h), n-1)$ 

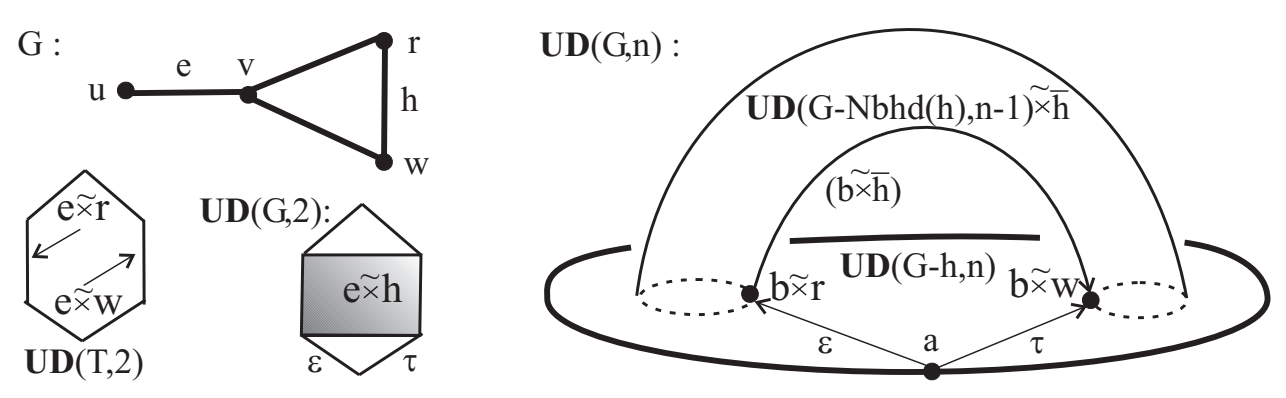

Figure 9: Adding an edge $h$ creating cycles

splits into subspaces $\mathbf{U D}_{J}(G-\operatorname{Nbhd}(h), n-1)$ indexed by $J=\left(j_{1}, \ldots, j_{k}\right)$ with nonnegative integer entries such that $j_{1}+\cdots+j_{k}=n-1$; see similar notations before Proposition 3.2. Fix $a \in \mathbf{U D}(G-h, n)$ and $b_{J} \in \mathbf{U D}_{J}(G-\operatorname{Nbhd}(h), n-1)$.

Denote by $\left(b_{J} \tilde{\times} h\right) \subset \mathbf{U D}(G, n)$ the motion such that one robot goes along the edge $h$ from $r$ to $w$, while the other robots remain fixed at the base configuration $b_{J} \in \mathbf{U D}_{J}(G-\operatorname{Nbhd}(h), n-1)$; see the right picture of Figure 9 . In the case $k=1$, when $G-\operatorname{Nbhd}(h)$ is connected, one can skip the index $J$. Take paths $\varepsilon_{J}, \tau_{J}$ going from $a$ to $b_{J} \tilde{\times} r, b_{J} \tilde{\times} w$, respectively, in $\mathbf{U D}(G-h, n)$; see Algorithm 4.3. Then $\varepsilon_{J}\left(b_{J} \tilde{\times} h\right) \tau_{J}^{-1}$ is a loop with the base point $a$ in the space $\mathbf{U D}(G, n)$.

Proposition 3.6 (Adding an edge $h$ creating cycles). Given the presentations

$$
\pi_{1}(\mathbf{U D}(G-h, n))=\langle\boldsymbol{\alpha} \mid \boldsymbol{\rho}\rangle \quad \text { and } \quad \pi_{1}\left(\mathbf{U D}_{J}(G-\operatorname{Nbhd}(h), n-1)\right)=\left\langle\boldsymbol{\beta}_{J} \mid \boldsymbol{\lambda}_{J}\right\rangle,
$$

the group $\pi_{1}(\mathbf{U D}(G, n))$ is generated by $\boldsymbol{\alpha}, \varepsilon_{J}\left(b_{J} \tilde{\times} h\right) \tau_{J}^{-1}$ subject to $\boldsymbol{\rho}=1$ and

$$
\varepsilon_{J}\left(\boldsymbol{\beta}_{J}\left\{x_{n}=r\right\}\right) \varepsilon_{J}^{-1}=\left(\varepsilon_{J}\left(b_{J} \tilde{\times} h\right) \tau_{J}^{-1}\right) \cdot\left(\tau_{J}\left(\boldsymbol{\beta}_{J}\left\{x_{n}=w\right\}\right) \tau_{J}^{-1}\right) \cdot\left(\varepsilon_{J}\left(b_{J} \tilde{\times} h\right) \tau_{J}^{-1}\right)^{-1} \text {. }
$$

Proof. Each of the subspaces $\mathbf{U D}_{J}(G-\operatorname{Nbhd}(h), n-1) \tilde{\times} \bar{h}$ meets the subspace $\mathbf{U D}(G-h, n)$ along $\mathbf{U D}_{J}(G-\operatorname{Nbhd}(h), n-1) \tilde{\times} r, \mathbf{U D}_{J}(G-\operatorname{Nbhd}(h), n-1) \tilde{\times} w$.

First add to each subspace $\operatorname{UD}_{J}(G-\operatorname{Nbhd}(h), n-1) \tilde{x} \bar{h}$ the union of the paths $\varepsilon_{J} \cup \tau_{J}$ as shown in Figure 9, where indices $J$ are skipped for simplicity. The fundamental group of $\left(\mathbf{U D}_{J}(G-\operatorname{Nbhd}(h), n-1) \tilde{\times} \bar{h}\right) \cup\left(\varepsilon_{J} \cup \tau_{J}\right)$ is isomorphic to the free product of $\mathbf{B}(G-\operatorname{Nbhd}(h), n-1)$ and $\mathbb{Z}$ generated by the loop $\varepsilon_{J}\left(b_{J} \tilde{\times} h\right) \tau_{J}^{-1}$. Second, add to $\mathbf{U D}(G-h, n)$ each union $\left(\mathbf{U D}_{J}(G-\operatorname{Nbhd}(h), n-1) \tilde{\times} \bar{h}\right) \cup\left(\varepsilon_{J} \cup \tau_{J}\right)$. The intersection of the spaces attached above is the union over all $J$ of the subspaces

$$
\left(\mathbf{U D}_{J}(G-\operatorname{Nbhd}(h), n-1) \tilde{\times} r\right) \cup\left(\varepsilon_{J} \cup \tau_{J}\right) \cup\left(\mathbf{U D}_{J}(G-\operatorname{Nbhd}(h), n-1) \tilde{\times} w\right) .
$$

Each space above is homotopically a wedge of two copies of $\mathbf{U D}_{J}(G-\operatorname{Nbhd}(h), n-$ 1). By Seifert-van Kampen Theorem 3.1, express the loops $\varepsilon_{J}\left(\boldsymbol{\beta}_{J}\left\{x_{n}=r\right\}\right) \varepsilon_{J}^{-1}$ and $\tau_{J}\left(\boldsymbol{\beta}_{J}\left\{x_{n}=w\right\}\right) \tau_{J}^{-1}$ generating the fundamental group of the intersection in terms of the loops from $\mathbf{U D}(G-h, n)$ and $\left(\mathbf{U D}_{J}(G-\operatorname{Nbhd}(h), n-1) \tilde{\times} \bar{h}\right) \cup\left(\varepsilon_{J} \cup \tau_{J}\right)$. In the latter space these loops are conjugated by $\varepsilon_{J}\left(b_{J} \tilde{\times} h\right) \tau_{J}^{-1}$ as required, i.e., homotopic through the subspace $\mathbf{U D}_{J}(G-\operatorname{Nbhd}(h), n-1) \tilde{\times} \bar{h}$.

If the vector $\boldsymbol{\beta}$ is empty, i.e., the groups $\pi_{1}\left(\mathbf{U D}_{J}(G-\operatorname{Nbhd}(h), n-1)\right)$ are trivial, then no new relations are added in Proposition 3.6. 


\section{Computing graph braid groups}

Step-by-step instructions of Algorithm 1.5 are based on the technical propositions from Section 3 and the auxiliary algorithms from Subsection 4.1. Proposition 4.8 extends the result about 2-point braid groups of graphs with only disjoint cycles $[\mathbf{6}$, Theorem 5.6] to a wider class of graphs including all light planar graphs.

\subsection{A motion planning algorithm in the unordered case}

Proposition 3.2 requires a collision free motion connecting two configurations of $n$ robots. Take a connected graph $G$ and number its vertices. Let us work with discrete configuration spaces assuming that at every discrete time moment all robots are at vertices of a graph $G$. So in one step any robot can move to an adjacent vertex if it is not occupied. The output contains positions of all robots at every moment.

To describe planning Algorithm 4.3 introduce auxiliary definitions and searching Algorithms 4.1 and 4.2. A robot $x_{i} \in G$ is called extreme in a given configuration $\left(x_{1}, \ldots, x_{n}\right) \in \mathbf{O D}(G, n)$ if the remaining robots are in one connected component of $G-\left\{x_{i}\right\}$. One configuration may have several extreme robots, e.g., on a segment there are always two extreme robots, while on a circle every robot is extreme.

Algorithm 4.1. If a graph $G$ has $l$ edges, then there is an algorithm of complexity $O(n l)$ to find an extreme robot in a configuration $\left(x_{1}, \ldots, x_{n}\right) \in \mathbf{O D}(G, n)$.

Proof. For a robot $x_{i}$, visit all vertices from a connected component of $G-\left\{x_{i}\right\}$ remembering the robots that were seen. If not all robots were seen, then $x_{i}$ is not extreme, so choose a robot $x_{j}$ that was visited in a component of $G-\left\{x_{i}\right\}$. If $x_{j}$ is extreme among the robots in the closure of this component $G-\left\{x_{i}\right\}$, then $x_{j}$ is extreme in the given configuration $\left(x_{1}, \ldots, x_{n}\right) \in \mathbf{O D}(G, n)$. Hence one inevitably finds an extreme robot, which requires not more than $l$ steps for any candidate.

A robot $x_{j}$ is a neighbour of a robot $x_{i}$ if a shortest path from $x_{i}$ to $x_{j}$ has the minimal number of edges among all shortest paths from $x_{i}$ to robots $x_{k}$ for $k \neq i$. For $n$ robots on a segment each of the two extreme robots has a unique neighbour, while on a circle each robot has two neighbours. A shortest path to a neighbour does not contain other robots, i.e., the corresponding motion is collision free.

Algorithm 4.2. If a connected graph $G$ has $l$ edges, then there is an algorithm of complexity $O(l)$ to find a shortest path from a robot $x_{i}$ to one of its neighbour $x_{j}$ in a given configuration of unordered robots $\left(x_{1}, \ldots, x_{n}\right) \in \mathbf{U D}(G, n)$.

Proof. One travels on $G$ in a 'spiral' way starting from $x_{i}$. So first visit all vertices adjacent to $x_{i}$ and check if there is another robot $x_{j}$ at one of them, which can be a neighbour of $x_{i}$. If not, then repeat the same procedure recursively for all these adjacent vertices. In total, one passes through not more than $l$ edges of $G$.

Algorithm 4.3. If a connected graph $G$ has $l$ edges, then there is an algorithm of complexity $O\left(n^{2}(l+n)\right)$ that, given any two configurations of $n$ unordered robots in $G$, finds a path in the space $\mathbf{U D}(G, n)$ between the given configurations.

Proof. Assume that all robots are at vertices of degree 2. Otherwise subdivide edges of the graph $G$ and move a robot to a closest vertex of degree 2. This subdivision 
increases the number $l$ of edges by not more than $n$ to $l+n$. The initial (and final) configuration of robots is considered as an array of vertices where the $n$ robots are located. During each elementary move, only one robot goes to an adjacent vertex. The resulting configuration is represented by a new array of positions.

Step 1. Using Algorithm 4.1 of complexity $O(n(l+n))$, find an extreme robot in the collection of $2 n$ given positions (initial and final together) ordered arbitrarily.

Step 2. Assume that the found extreme robot, say $y_{n}$, is from the final configuration, otherwise swap the roles of initial and final positions. Using Algorithm 4.2 of complexity $O(l+n)$, find a shortest path from $y_{n}$ to its neighbour, say $x_{n}$, from the initial configuration. Then safely move $x_{n}$ towards $y_{n}$ along the shortest path without any collisions and keeping fixed all other robots from the initial configuration.

Step 3. In the graph $G$ remove the robot $y_{n}$ located at a vertex of degree 2 and all open edges attached to $y_{n}$. This removal reduces the problem to a smaller graph with $n-1$ robots. The new graph remains connected since the robot $y_{n}$ was extreme. Return to Step 1 and apply the recursion $n-1$ times, which gives $O\left(n^{2}(l+n)\right)$ operations.

In Algorithm 4.3 the quadratic complexity in the number of robots seems to be asymptotically optimal, because avoiding collisions between $n$ robots should involve some analysis of their pairwise positions. Another quadratic algorithm for checking the non-trivial topological property of basic embeddability of any finite graphs into a product of small graphs was designed in [10]. One more algorithm of linear complexity was found to verify whether a combinatorial code (a Gauss paragraph of several words) encodes a classical link in 3-space [11]. Finally, Algorithm 1.5 is deterministic up to ordering edges of $G$ and choosing an extreme robot at every stage.

\section{Step-by-step instructions of Algorithm 1.5}

Step 1. To write down a presentation of the group $\mathbf{B}(G, n)$ for an arbitrary connected graph $G$, start from from $n$ robots on a segment subdivided into $n-1$ subsegments. Then the configuration space $\mathbf{U D}([0,1], n)$ is a single point, so $\mathbf{B}([0,1], n)$ is trivial.

Step 2. Fix a plan to construct the given graph $G$ by adding edges to $[0,1]$.

Step 3. If a hanging edge is added to a vertex of degree at least 2, then follow the rules of Proposition 3.2 to update the presentation of the braid group of the already constructed graph. If one needs a motion to connect two configurations of $n$ robots, then follow the steps of motion planning Algorithm 4.3.

Step 4. If a hanging edge is added to a vertex of degree 1, then follow the rules of Proposition 3.4 to update the presentation of the current graph braid group.

Step 5. If one adds an edge that creates a new cycle, then apply Proposition 3.6.

Every generator in the resulting group presentation is encoded by a list of successive configurations that show where the robots are located at every discrete moment.

\subsection{Two-point braid groups of graphs in the unordered case}

The first part of Lemma 4.4 without computing the rank was obtained by the global approach of Abrams [1, Corollary 3.8]. The second part was claimed in [4, Theorems 9, 10]. Both parts easily follow from our local step-by-step computations. 
Lemma 4.4. For any tree $H$, the braid group $\mathbf{B}(H, 2)$ is free and has the rank $\sum(\operatorname{deg} v-1)(\operatorname{deg} v-2) / 2$, where the sum is over all vertices of degree at least 3 .

Proof. Induction on the number of edges of $H$. The base $H \approx[0,1]$ is trivial. In the inductive step notice that trees are contractible. Hence their fundamental groups are trivial and for $n=2$ the vectors $\boldsymbol{\beta}, \boldsymbol{\gamma}, \boldsymbol{\lambda}, \boldsymbol{\mu}$ (with indices) are empty in Propositions 3.2 and 3.4. The vectors $\boldsymbol{\rho}$ are also empty, because they can only come from 2-point braid groups of smaller trees. So the braid group $\mathbf{B}(H, 2)$ is free. In the case $n=2$ the multiple index $J$ in Proposition 3.2 degenerates to a single index $j=1, \ldots, \operatorname{deg} v-1$. Then the only generators of $\mathbf{B}(H, 2)$ are $\delta_{1} \delta_{j}^{-1}, j=2, \ldots, \operatorname{deg} v-1$. In total, one gets

$$
1+2+\cdots+(\operatorname{deg} v-2)=(\operatorname{deg} v-1)(\operatorname{deg} v-2) / 2
$$

generators after attaching all edges to each vertex $v$ of degree $\operatorname{deg} v$.

The Kuratowski graphs $K_{5}, K_{3,3}$ in Figure 2 do not satisfy Lemma 4.5. Indeed, the complement to the neighbourhood of any edge $h \in K_{5}$ ( $h \in K_{3,3}$, respectively) is the triangular (rectangular, respectively) cycle intersecting any cycle $C \supset h$.

Lemma 4.5. Any light planar graph can be constructed from a tree by adding edges as follows: an open edge $h$ added to the new graph $G$ creates a cycle $C$ not meeting any cycle from $G-\operatorname{Nbhd}(h)$ having all its cycles in one connected component.

Proof. A planar connected graph $G$ is light if any cycle $C \subset G$ has an edge $h$ such that all cycles from $G-\bar{h}$ (or, equivalently, $G-\operatorname{Nbhd}(h)$ ) do not meet $C$. For a given light planar graph $G$, take any cycle $C$ and the corresponding edge $h$. The subgraph $G-h$ is light planar, because $G-h$ has fewer cycles satisfying the same condition.
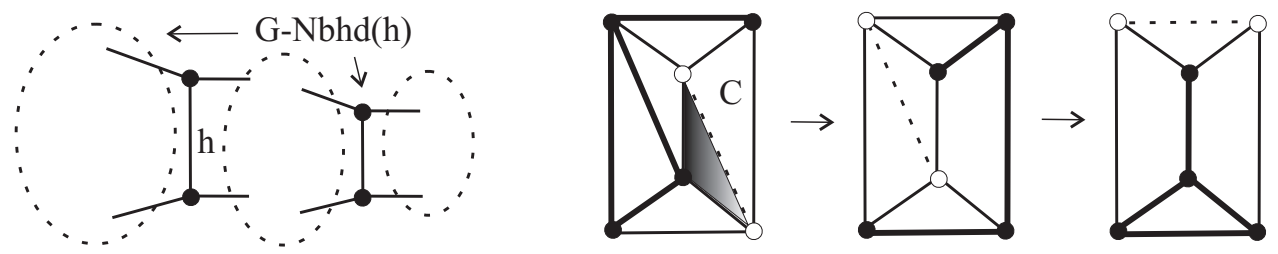

Figure 10: Choosing an edge $h$ and a cycle $C \supset h$ in Lemma 4.5

One can assume that all cycles of the subgraph $G-\operatorname{Nbhd}(h)$ are in one connected component. Otherwise, choose another cycle from a component of $G-\operatorname{Nbhd}(h)$ with a smaller number of edges; see the left-hand side picture of Figure 10, and so on until one finds a cycle with an edge $h$ such that $G-\operatorname{Nbhd}(h)$ has all its cycles in one component. Remove edges one-by-one until the graph becomes a tree. Then the original graph $G$ can be reconstructed by reversing the procedure above.

The construction from Lemma 4.5 is also applicable to some non-light planar graphs. The right picture of Figure 10 shows three stages of such a construction, where the closed edge $\bar{h}$ is dashed and the corresponding subgraph $G-\operatorname{Nbhd}(h)$ has thick edges. The biggest graph fails to be light planar because of the cycle bounding the grey triangle. For the same graph and the dashed edge $h$, one can choose another cycle $C$ that does not meet the only (triangular) cycle from $G-\operatorname{Nbhd}(h)$. Lemma 4.5 
implies that Corollary 1.6 for unordered robots is a particular case of more technical Proposition 4.8, which holds for all graphs constructed as described above.

Corollary 4.6. The A-graph in the left-hand side picture of Figure 11 below has the braid group $\mathbf{B}(A, 2) \cong F_{3}$, the free group with three generators.

Proof. Three proofs are given below to illustrate different approaches.

1. The $A$-graph is obtained from the graph $G$ in the top left picture of Figure 9 in Example 3.5 by adding the hanging edge $h_{1}$ to the vertex $w$ of degree 2. The complement $A-\operatorname{Nbhd}\left(h_{1}\right) \approx[0,1]$ is connected. Hence one can skip the multiple index $J$ and apply Proposition 3.2 to the free group $\mathbf{B}(G, 2) \cong F_{2}$. One adds no relations and one generator $\delta_{1} \delta_{2}^{-1}$ since $\operatorname{deg} w=3$ in the $A$-graph, so $\mathbf{B}(A, 2) \cong F_{3}$.

2. The same $A$-graph can also be obtained by adding the open edge $h_{2}$ to the graph $H$ in the top left picture of Figure 8; see Example 3.3. One of the endpoints of $h_{2}$ is the vertex $u \in H$ of degree 2 , and the other one is the hanging vertex $v_{1}$. Apply Proposition 3.6 to compute $\mathbf{B}(A, 2)$ from $\mathbf{B}(H, 2) \cong \mathbb{Z}$. The complement $A-\operatorname{Nbhd}\left(h_{2}\right)$ is not connected and consists of the closed hanging edge $\bar{g}$ and the remaining hanging vertex $v_{2} \in H$. Then the multiple index $J$ takes only two values $(1,0)$ and $(0,1)$ in Proposition 3.6, i.e., one adds no relations and two new generators of the form $\varepsilon_{J}(b \tilde{\times} h) \tau_{J}^{-1}$ to $\mathbf{B}(H, 2) \cong \mathbb{Z}$. Hence one gets the free group $\mathbf{B}(A, 2) \cong F_{3}$ as expected.

3 . Finally the $A$-graph can be obtained from the segment $[0,1]$ with vertices 0 , $1 / 4,1 / 2,3 / 4,1$ by adding an open edge $h_{3}$ at $1 / 4,3 / 4$. Then the complement $A-\operatorname{Nbhd}\left(h_{3}\right)$ consists of three vertices $0,1 / 2,1 \in[0,1]$. Hence Proposition 3.6 adds three generators without relations to the group $\mathbf{B}([0,1], 2)=1$, i.e., $\mathbf{B}(A, 2)$ $\cong F_{3}$.
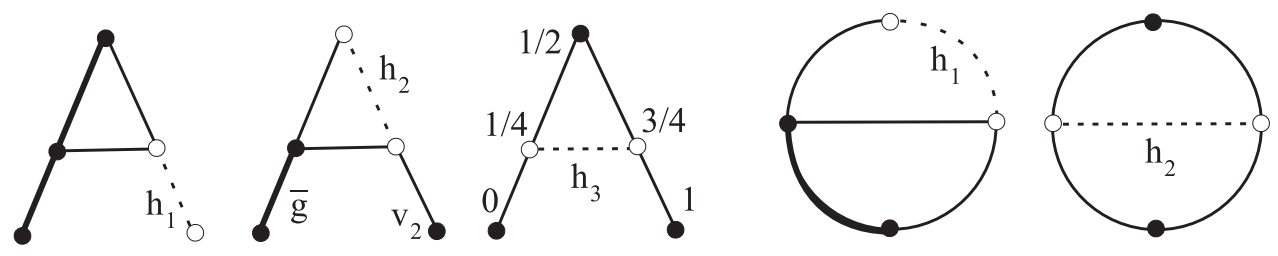

Figure 11: Choosing different edges in the $A$-graph and $\theta$-graph

Corollary 4.7. The $\theta$-graph in the right-hand side picture of Figure 11 above has the braid group $\mathbf{B}(\theta, 2) \cong F_{3}$, the free group with three generators.

Proof. Two proofs are given below to illustrate different approaches:

1. The $\theta$-graph is obtained from the graph $G$ in the top left-hand side picture of Figure 9 in Example 3.5 by adding an open edge $h_{1}$ connecting the vertices $u, w \in G$. Compute $\mathbf{B}(\theta, 2)$ from $\mathbf{B}(G, 2) \cong F_{2}$ by using Proposition 3.6. Then $\theta-\operatorname{Nbhd}\left(h_{1}\right)$ is a single edge. No relations and one generator are added, so one gets $\mathbf{B}(\theta, 2) \cong F_{3}$. 
2. The $\theta$-graph can also be obtained from a circle $S^{1}$ with two pairs of opposite vertices by adding an open horizontal diameter $h_{2}$ at one of these pairs. Then the complement $\theta-\operatorname{Nbhd}\left(h_{2}\right)$ is the other pair of the opposite vertices. Hence the multiple index $J$ in Proposition 3.6 takes only two values $(1,0)$ and $(0,1)$. This adds no relations and two generators to $\mathbf{B}\left(S^{1}, 2\right) \cong \mathbb{Z}$, i.e., $\mathbf{B}(\theta, 2) \cong F_{3}$ as expected.

Corollaries 4.6 and 4.7 agree with [9, Example 2.1] and [6, Example 5.2], respectively, based on the discrete Morse theory. The following result extends these computations to a wider class of graphs including all light planar graphs:

Proposition 4.8. Let a graph $G$ be constructed from a tree $T$ as in Lemma 4.5 by adding open edges $h_{1}, \ldots, h_{m}$, i.e., $G_{1}=T \cup h_{1}, G_{2}=G_{1} \cup h_{2}, \ldots, G=G_{m-1} \cup h_{m}$. Let $k_{j}$ be the number of connected components of $G_{j}-\operatorname{Nbhd}\left(h_{j}\right)$, where $G_{m}=G$.

The braid group $\mathbf{B}(G, 2)$ has a presentation with

$$
\sum_{j=1}^{m} k_{j}+\sum(\operatorname{deg} v-1)(\operatorname{deg} v-2) / 2
$$

generators subject to commutator relations, where the second sum is over all vertices $v \in T$ of degree at least 3 in the tree T. A geometric description follows:

1. At each vertex $v \in G$ fix an edge $e_{0}$. For any unordered pair of other edges $e_{i}, e_{j}$ at the same vertex $v, j=1, \ldots, \operatorname{deg} v-1$, one generator of $\mathbf{B}(G, 2)$ swaps two robots in the tripod $e_{0} \cup e_{i} \cup e_{j}$ by using the collision free motion shown in Figure 1.

2. The remaining $\sum_{j=1}^{m} k_{j}$ generators of $\mathbf{B}(G, 2)$ represent motions when one robot remains in a connected component of $G_{j}-\operatorname{Nbhd}\left(h_{j}\right)$, and the other robot moves without collisions along a cycle $\hat{h}_{j}$ containing the open edge $h_{j}$ chosen above.

3. Each relation says that motions of two robots along disjoint cycles commute.

Proof. Computing the 2-point braid group $\mathbf{B}(G, 2)$ by Subdivision Theorem 1.4, assume that $G$ has no loops and multiple edges after removing extra trivial vertices of degree 2. Induction on the first Betti number $m$. Base $m=0$ is Lemma 4.4, where every generator $\delta_{1} \delta_{j}^{-1}$ coming from Proposition 3.2 is represented by a loop that swaps two robots near a vertex of degree at least 3 as shown in Figure 1.

In the inductive step from $m-1$ to $m$, for an open edge $h \subset G$ from Lemma 4.5, let us show how a presentation of $\mathbf{B}(G, 2)$ differs from a presentation of $\mathbf{B}(G-h, 2)$ satisfying the conditions by the inductive hypothesis. Proposition 3.6 adds motions such that one of the two robots moves along the newly added edge $h$, while the other robot remains in a connected component of $G-\operatorname{Nbhd}(h)$, whose index is encoded by the place of 1 in the $k_{m}$-tuple index $J$ of the form $(0, \ldots, 0,1,0 \ldots, 0)$.

The relations $\boldsymbol{\lambda}_{J}$ are trivial since $n=2$ and fundamental groups of graphs are free. By Lemma 4.5 the complement $G-\operatorname{Nbhd}(h)$ has all its cycles in one connected component. Hence the generators $\boldsymbol{\beta}_{J}$ of $\pi_{1}\left(\mathbf{U D}_{J}(G-\operatorname{Nbhd}(h), 1)\right)$ are non-trivial only for one value of the multiple index $J$. As required by the formula, add $k_{m}$ generators $\varepsilon_{J}\left(b_{J} \tilde{\times} h\right) \tau_{J}^{-1}$ that, for the single value of $J$, conjugate the loops $\varepsilon_{J}\left(\boldsymbol{\beta}_{J}\left\{x_{2}=r\right\}\right) \varepsilon_{J}^{-1}$ and $\tau_{J}\left(\boldsymbol{\beta}_{J}\left\{x_{2}=w\right\}\right) \tau_{J}^{-1}$. Geometrically, $\varepsilon_{J}\left(b_{J} \tilde{\times} h\right) \tau_{J}^{-1}$ represents a motion when one robot stays away from the other robot completing a cycle $\hat{h} \supset h$. 
It remains to show that the loops $\left(\boldsymbol{\beta}_{J}\left\{x_{2}=r\right\}\right)$ and $\left(\boldsymbol{\beta}_{J}\left\{x_{2}=w\right\}\right)$ are homotopic, i.e., the new relator is a commutator. Take the cycle $C \supset h$ from the construction of Lemma 4.5. Since $C$ does not meet all cycles from $G-\operatorname{Nbhd}(h)$, then one can move one robot along $C-h$ from $r$ to $w$ without collisions with the other robot is moving along the loops $\boldsymbol{\beta}_{J}$. These loops generate the fundamental group of the single non-contractible component of $G-\operatorname{Nbhd}(h)$. One gets a free homotopy from

$$
\left(\boldsymbol{\beta}_{J}\left\{x_{2}=r\right\}\right) \text { to }\left(\boldsymbol{\beta}_{J}\left\{x_{2}=w\right\}\right)=\left(b_{J} \tilde{\times}(C-h)\right) \cdot\left(\boldsymbol{\beta}_{J}\left\{x_{2}=r\right\}\right) \cdot\left(b_{J} \tilde{\times}(C-h)\right)^{-1} \text {. }
$$

During the motion $\left(b_{J} \tilde{\times}(C-h)\right)$, one robot is fixed at $b_{J} \in G-\operatorname{Nbhd}(h)$, while the other moves along $C-h$ avoiding all cycles of $G-\operatorname{Nbhd}(h)$. In Proposition 3.6 choose the path $\tau_{J}$ from $a$ to $b_{J} \tilde{\times} w$ in $\mathbf{U D}(G-h, 2)$ so that $\tau_{J}=\varepsilon_{J} \cdot\left(b_{J} \tilde{\times}(C-h)\right)$. Then the loops $\varepsilon_{J}\left(\boldsymbol{\beta}\left\{x_{2}=r\right\}\right) \varepsilon_{J}^{-1}$ and $\tau_{J}\left(\boldsymbol{\beta}_{J}\left\{x_{2}=w\right\}\right) \tau_{J}^{-1}$ are homotopic with the fixed base configuration $a \in \mathbf{U} \mathbf{D}(G-h, 2)$.

The following result agrees with [6, Example 5.4]:

Corollary 4.9. The $\oplus$-graph in the left-hand side picture of Figure 12 below has the braid group $\mathbf{B}(\oplus, 2) \cong F_{5}$, the free group with five generators.

Proof. The open edge $h_{0} \subset \oplus$ can not appear in the construction of Lemma 4.5 since all cycles of $\oplus$ through $h_{0}$ meet the triangle $\oplus-\operatorname{Nbhd}\left(h_{0}\right)$ at the central vertex.

The vertical radius $h_{1}$ is allowed by Lemma 4.5 since $\oplus-\operatorname{Nbhd}\left(h_{1}\right) \approx[0,1]$ is the lower half-circle. Then Proposition 3.6 implies that $\mathbf{B}(\oplus, 2)$ is the free product of $\mathbb{Z}$ and $\mathbf{B}\left(\oplus-h_{1}, 2\right)$. Applying Lemma 4.5 to the subgraph $\oplus-h_{1}$, choose the remaining vertical radius $h_{2}$ such that $\left(\oplus-h_{1}\right)-\operatorname{Nbhd}\left(h_{2}\right) \approx[0,1]$ is the upper halfcircle. By Proposition 3.6 the group $\mathbf{B}\left(\oplus-h_{1}, 2\right)$ is the free product of $\mathbb{Z}$ and the group $\mathbf{B}\left(\oplus-\left(h_{1} \cup h_{2}\right), 2\right)=\mathbf{B}(\theta, 2) \cong F_{3}$ by Corollary 4.7 , hence $\mathbf{B}(\oplus, 2) \cong F_{5}$.
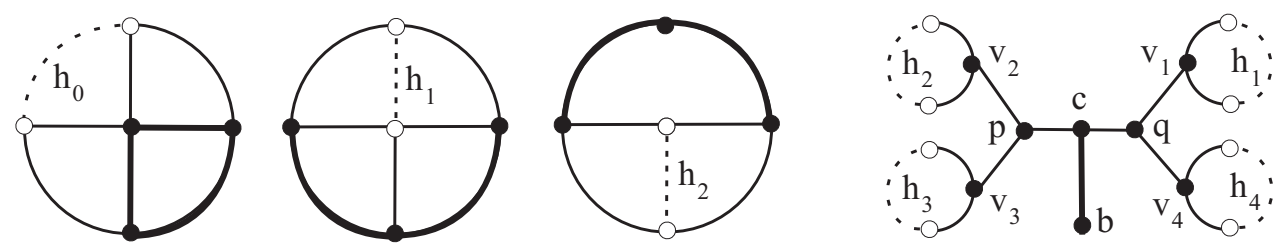

Figure 12: Choosing different edges in the $\oplus$-graph and tree with balloons

Corollary 4.10. The graph $G$ in the right-hand side picture of Figure 12 has the braid group $\mathbf{B}(G, 2)$ that has a presentation with 11 generators and six commutator relations.

Proof. The graph $G$ has four loops $f_{1}, f_{2}, f_{3}, f_{4}$ attached at the vertices $v_{1}, v_{2}, v_{3}, v_{4}$, respectively. Computing $\mathbf{B}(G, 2)$ by Theorem 1.4, one can subdivide each simple loop $f_{j}$ into three subedges. Denote by $h_{j} \subset f_{j}$ the middle subedge not containing $v_{j}$. Then $G-\operatorname{Nbhd}\left(h_{j}\right)$ is obtained from $G$ by removing the simple loop $f_{j}$, but keeping $v_{j}$. The group $\mathbf{B}(G, 2)$ will be computed by removing the open edges $h_{j}$.

By Lemma 4.4 for $T=G-\left(h_{1} \cup h_{2} \cup h_{2} \cup h_{3}\right)$, the group $\mathbf{B}(T, 2)$ is free and is generated by the seven motions $s(v)$ swapping two robots around the seven vertices 
of degree 3 in $T$. Let $\varepsilon_{1}$ be the natural path from the base configuration $b \tilde{\times} c$ to $b \tilde{\times} v_{1}$. Before applying Proposition 3.6, gradually move the endpoints of $h_{1}$ to the adjacent vertex $v_{1}$, then the path $\tau_{1}$ coincides with $\varepsilon_{1}$. Attaching the open edge $h_{1}$ adds no relations and the generator $e_{1}=\varepsilon_{1}\left(b \tilde{\times} f_{1}\right) \varepsilon_{1}^{-1}$ representing the motion when one robot stays at $b$, while the other robot goes around $f_{1}$. Attaching the next open edge $h_{2}$ adds the similar generator $e_{2}=\varepsilon_{2}\left(b \tilde{\times} f_{2}\right) \varepsilon_{2}^{-1}$ and one relation saying that $e_{2}$ commutes with $\varepsilon_{2}\left(f_{1}\left\{x_{2}=v_{2}\right\}\right) \varepsilon_{2}^{-1}$, where $\varepsilon_{2}$ is the natural path from $b \tilde{\times} c$ to $b \tilde{\times} v_{2}$. Geometrically, the last loop is conjugated to $e_{1}$ by the motion $s(c)$ swapping the robots around $c$, i.e., the robots go around $f_{1}, f_{2}$ without collisions: $\left[e_{2}, s(c) e_{1} s(c)^{-1}\right]=1$.

Attaching $h_{3}, h_{4}$ similarly adds the generators $e_{3}, e_{4}$ and $\left[e_{3}, s(c) e_{1} s(c)^{-1}\right]=1$, $\left[e_{3}, s(p) e_{2} s(p)^{-1}\right]=\left[e_{4}, s(q) e_{1} s(q)^{-1}\right]=\left[e_{4}, s(c) e_{2} s(c)^{-1}\right]=\left[e_{4}, s(c) e_{3} s(c)^{-1}\right]=1$.

Corollaries 4.9 and 4.10 show that Proposition 4.8 and general Algorithm 1.5 effectively compute braid groups of graphs by using known results without starting from scratch. Generalising Sections 3 and 4 to the case of ordered robots is left to the reader.

\section{References}

[1] A. Abrams, Configuration spaces and braid groups of graphs, Ph.D. thesis, 2000, UC Berkeley, available at http://www.math.uga.edu/ abrams/ research.

[2] C. Adams and R. Franzosa, Introduction to topology: pure and applied, Pearson Prentice Hall, Upper Saddle River, NJ, 2008.

[3] R. Crowell and R. Fox, Introduction to knot theory, Springer-Verlag, New York, 1963.

[4] M. Farber, Collision free motion planning on graphs, in Algorithmic foundations of robotics VI, Utrecht/Zeist, 2004, MPIM2004-87.

[5] D. Farley and L. Sabalka, Discrete Morse theory and graph braid groups, Algebraic and Geometric Topology 5 (2005), 1075-1109.

[6] D. Farley and L. Sabalka, Presentations of graph braid groups, arXiv: 0907.2730

[7] R. Forman, Morse theory for cell complexes, Adv. Math. 134 (1998), no. 1, 90-145.

[8] R. Ghrist, Configuration spaces and braid groups on graphs in robotics, in Knots, braids, and mapping class groups-papers dedicated to Joan S. Birman (New York, 1988), AMS/IP Stud. Adv. Math. 24 (2000) 29-40, AMS, Providence, RI, 29-40.

[9] J.H. Kim, K.H. Ko and H.W. Park, Graph braid groups and right-angled Artin groups, Trans. Amer. Math. Soc. 364 (2012), no. 1, 309-360.

[10] V. Kurlin, Basic embeddings into a product of graphs, Topology Appl. 102 (2000), no. 2, 113-137.

[11] V. Kurlin, Gauss paragraphs of classical links and a characterization of virtual link groups, Math. Proc. Cambridge Philos. Soc. 145 (2008), no. 1, 129-140. 
[12] P. Prue and T. Scrimshaw, Abrams's stable equivalence for graph braid groups, arXiv:0909.5511.

Vitaliy Kurlin vitaliy.kurlin@durham.ac.uk

Department of Mathematical Sciences, Durham University, Durham DH1 3LE, UK 